\title{
A Second Order Transition in a Neuron Grid
}

\author{
R. Thieberger ${ }^{1}$, A. Rabinovitch ${ }^{1}$, A. Vainer ${ }^{1}$, I. Aviram ${ }^{1}$, Y. Biton ${ }^{1}$ and D. Braunstein ${ }^{2}$ \\ 1. Physics Department, Ben-Gurion University of the Negev, Beer-Sheva 84105, Israel \\ 2. Sami Shamoon College of Engineering, Beer-Sheva 84100, Israel
}

\begin{abstract}
We perform a simple calculation of a classical cellular automata model based on two types of cells (neurons), excitatory and inhibitory ones, randomly distributed in a 2D space. The only varying parameter is the percentage of inhibitory neurons. Even under such simple conditions, we obtain a transition between two states (which we conjecture to be normal and epileptic). The difference between these states are manifested both in the neuronal activity amplitude and in their frequency. The amplitude changes are shown to be similar to those obtained in a tonic-clonic seizure.
\end{abstract}

Key words: Cellular automata, epilepsy, seizures, neurons.

\section{Introduction}

Epilepsy is a major brain malfunction inflicting millions of people worldwide. Although being under extensive investigation both theoretically and experimentally for a long period, real understanding of the basic causes of the phenomenon is still missing. Seizure initiation, maintenance and termination are attributed to many operating causes. Examples are the changes in synaptic effectiveness [1], ion concentrations [2], cation conductance [3], glutamate concentration [4], connection of different brain parts [5] and $\mathrm{pH}$ [6]. The model which we wish to present in this work is intimately connected to having in the brain two types of neurons: excitatory and inhibitory. The former when activated deliver to the neurons a positive signal while the second type deliver the command "not to". A receiving neuron counts the number of impinging "orders" from all neurons it is on their receiving end, and decides whether to function according to the difference between the numbers of the two types. If the number of excitatory received information is larger, it becomes operational and vice versa. In the healthy brain the ratio of the number of

Corresponding author: R. Thieberger, Ph.D., professor of physics, research fields: statistical physics, computational physics. excitatory to the inhibitory neurons is approximately 4:1 [7]. Most observations indicate that, during an epilepsy seizure, there is a drop in the inhibitory neutrons. In this paper we assume that this is a fact. It seems that the major reason is the reduction of GABA ( $\gamma$ Aminobutyric acid) [4] which effectively reduces inhibition by decreasing the transmission of information out of such neutrons.

There exist some complex dynamical models, e.g. [8, 9], which use the value of the connection between the inhibitory and excitatory neurons as a bifurcation parameter. A different approach for mathematical modelling of epileptic behavior is based on network theory. e.g. [10].

We would like to treat a dynamical model in order to try to understand the global spatiotemporal basic processes appearing in epilepsy. Such understanding cannot be provided by network methods, which disregard the special attribute. Therefore, we chose a model based on cellular automata (CA) built from such two types of cells, which is possibly the simplest model of the brain conditions.

Cellular automata were first studied by Ulam and von Neumann with the particular purpose of modelling biological self-reproduction (see: Von Neumann [11]). Our Cellular Automaton model consists of a regular grid of cells in two dimensions. Each cell can attain just 
three modes (see below). For each cell a set of cells called its "neighborhood" is defined. An initial state at "time $=0$ " is assigned to each cell. A new generation is created (advancing " $\mathrm{t}$ " by one) by changing the state of each cell according to some rule that is determined by the state of the cell and its neighbors. This change is applied to the whole grid simultaneously. There were just few attempts in the literature to use CA for our problem [12]. Our approach is more similar to the classical method.

\section{The Model}

Our model consists of a two dimensional array of cells (or "neurons"). Each cell can be either excitatory (E) or inhibitory (I). Both neutron types are distributed randomly in the array. The percentage of the I-neurons is a varying parameter. Each cell can reside in one of three modes: ready (D), Refractory (R) and operating (O). We consider as "neighborhood" both the nearest and next nearest cells of a given central cell. At every cycle each neuron changes according to the following two rules: (1) An O-mode changes into an R-mode, an $\mathrm{R}$ mode changes, after a prescribed (an input parameter) number of cycles, into a D-mode, (2) All O-cells of a neighborhood of a D-mode neutron are counted and we subtract from the sum of the E-neurons the sum of the I-neurons. If this is a positive number, we change the D-mode into an O-mode. Following a cycle (i.e. going over all neurons) we update the total number of neurons in mode $=\mathrm{O}$ and consider this number as the measure of electric signal emerging from the array.

\section{Calculations and Results}

In our calculations we assume periodic boundary conditions. We checked also the case of Dirichlet boundary conditions and did not find large differences. Our grid consists of $120 \times 120$ cells ("neurons"). We chose four cycles for the refractory value. The number of cycles was 12,000. For our Fourier analysis of the results we discarded the first 6,000 cycles. For each case we repeated the calculation five times, and took the average result, so as to eliminate the influence of the randomly chosen distribution of inhibitory neurons. These calculations were performed for many different percentages of inhibitory neurons. As was mentioned before, the total number of neurons which are in the state of firing is our measure of the electric signal. We will call this measure: $\rho_{e}$. Results are given in Fig. 1a. Here we show $\rho_{e}$ as a function of the percentage of inhibitory neurons $\left(\rho_{i}\right)$.

To see if our results are robust, we repeated our calculations using a much larger grid: $360 \times 360$ (i.e. Nine times as many neurons). In Fig. 1b we show the results, which do not differ much.

Viewing both of these figures we see that around $\rho_{i}=0.2$ we have a change in the slope of the line. We wish to examine this result. For this purpose, we calculate the Fourier transform for different percentage of inhibitory neurons. At $\rho_{i}=0.17$ we obtain a peak frequency at 43.9 (see Fig. 2a) whereas at $\rho_{i}=0.23$, the peak jumps to 34.7 (see Fig. 2c).

We make the conjecture that this is a transition between a normal state and an epileptic state. As a justification we mention that it is this $\rho_{i}$ that the normal state goes over to the epileptic state in a real system.

We show next the results of considering a normal state which goes over to an epileptic state and returns after some time back to the normal one. We assume that when the percentage of inhibitory neurons is 19 percent we are in the epileptic state, and when there is 23 percent of inhibitory neurons we are in the normal state. So we calculate $\rho_{e}$ vs. time when going from $\rho_{i}=0.23$ to $\rho_{i}=0.19$ and back. To improve our figure, we added another feature. We assume that during the epileptic state we have a certain amount of oscillations in the O-neurons (see [13]). The amount we took in our figure was one percent. In Fig. 3a, we compare the electric excitation as a function of time to those obtained by EEG adopted from Ref. [14] (Fig. 3b). It is seen that even such a simple model can repeat the actual seizure behavior. 

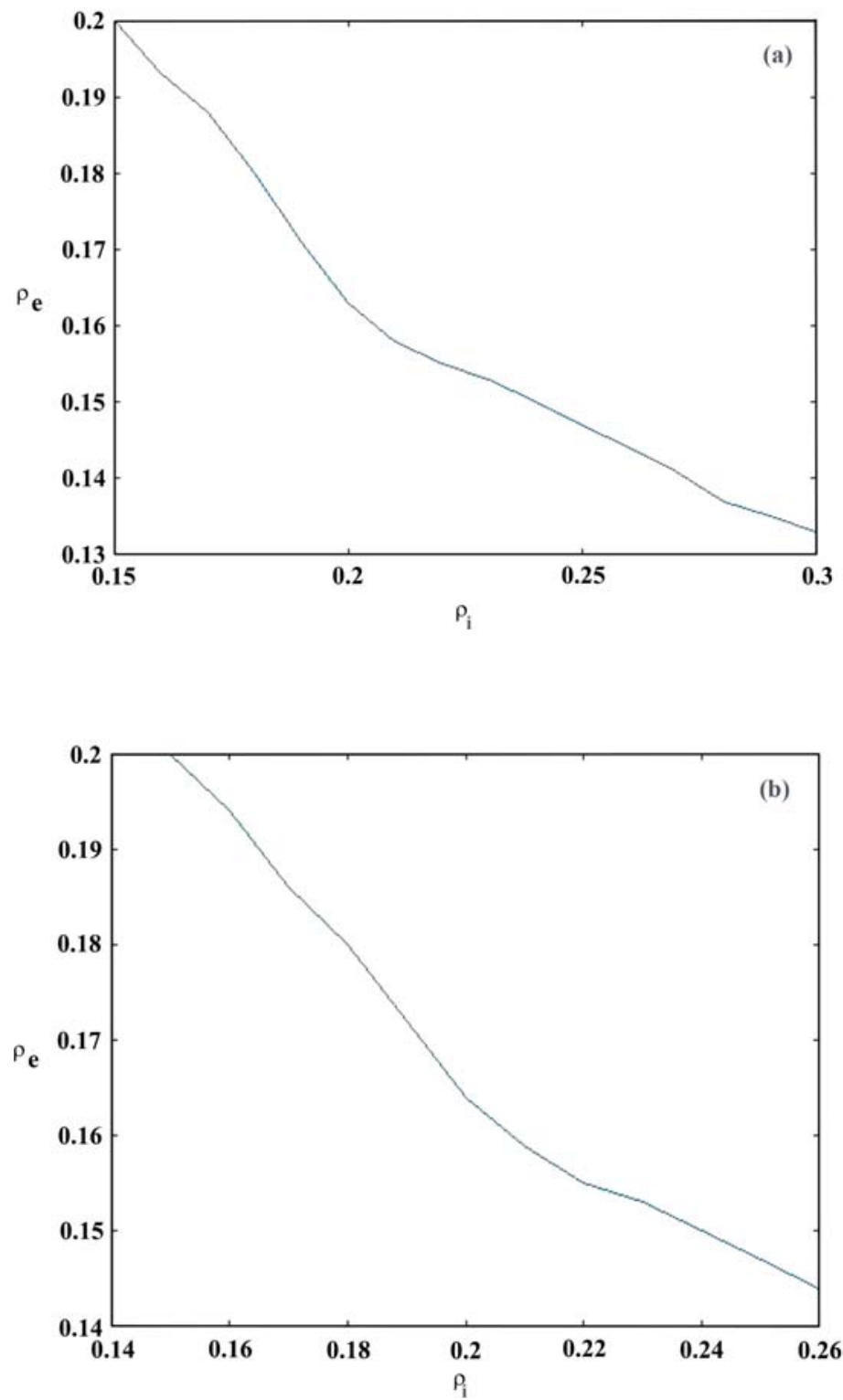

Fig. 1 Relative electric signal as a function of the inhibitory neurons fraction. (a) $120 \times 120$ grid; (b) $360 \times 360$ grid. 

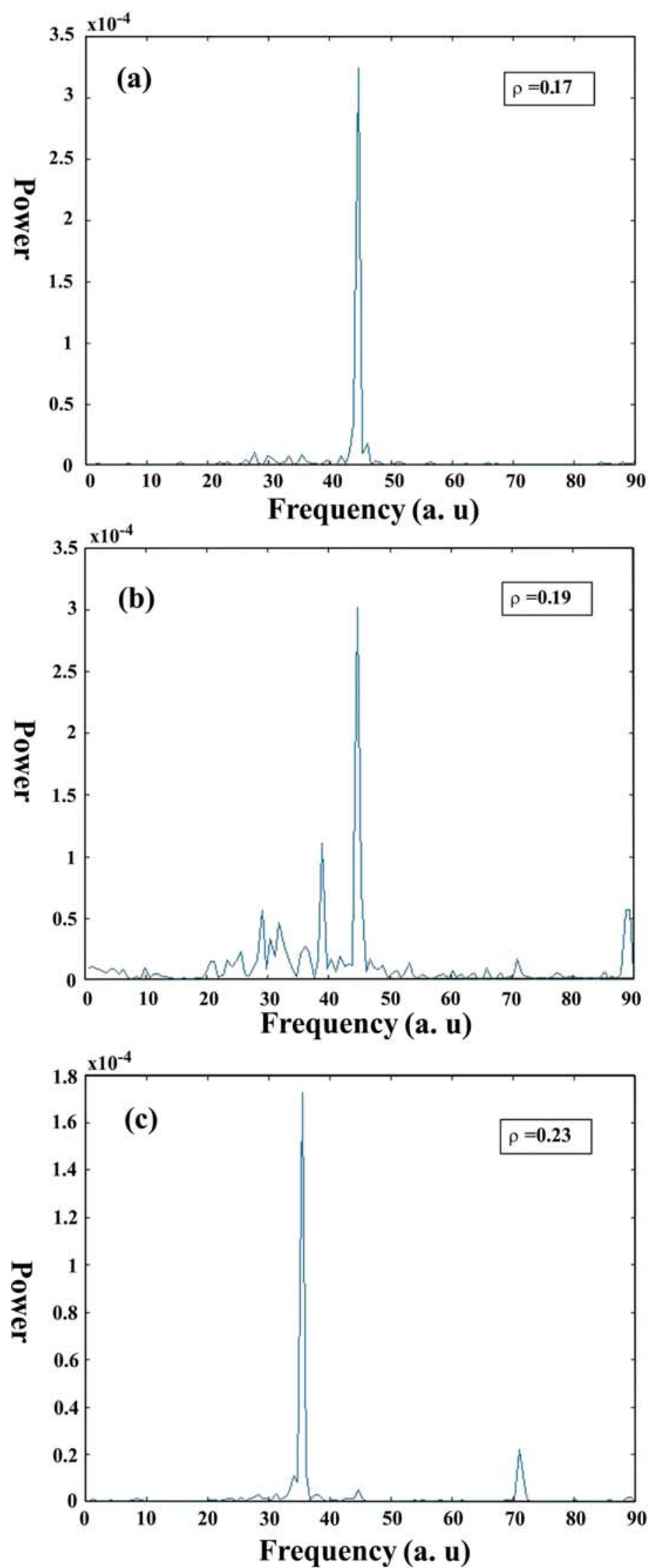

Fig. 2 Fourier power for: (a) 17\%, (b) 19\% and (c) $23 \%$ inhibitory neurons. 


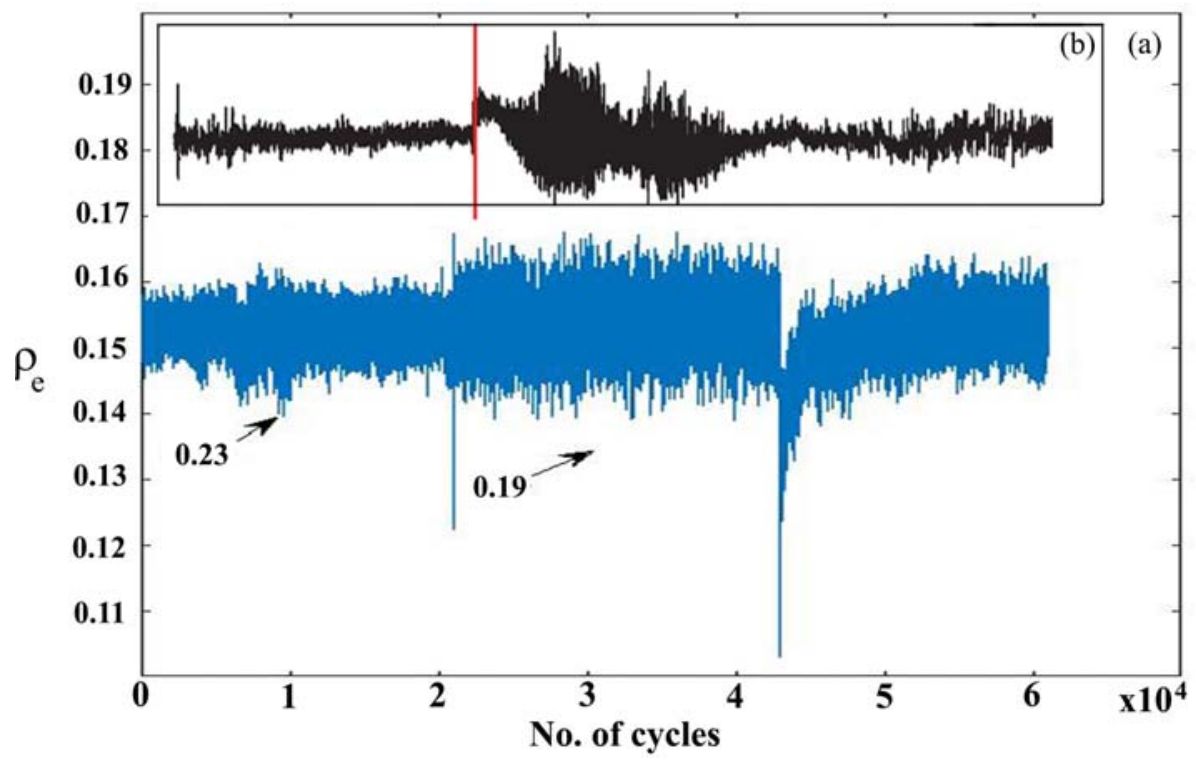

Fig. 3 (a) Relative electric signal for a passage from $23 \%$ to $19 \%$ and back to $23 \%$ of inhibitory neurons; (b) Actual EEG during an epileptic seizure (adapted from Ref. [14]).

\section{References}

[1] Froelich, F., Bazhenov, M., Timofeev, I., and Sejnowsky. T. J. 2005. "Mainenance and Termination of Neocortical Oscillations by Dynamic Modulation of Intrinsicand Synaptic Excitability.” Thalamus Relat Syst. 3: 147-56.

[2] Krishnan, G. P., and Bazhenov, M. 2011. "Ionic Dynamics Mediate Spontaneous Termination of Seizures and Postical Depression State.” J. Neurosci. 31: 8870-82.

[3] Bal, T., and McKormick, D. A. 1996. "What Stops Synchronized Talamocortical Oscilations?” Neuron 17: 297-308.

[4] Kullmann, D. M., and Semyanov. A. 2002. "Glutamatergic Modulation of GABA Signaling among Hipocampal Interneurons. Novel Mechanisms Regulating Hipocampal Excitability.” Epilepsia 43 (suppl. 5): 174-8.

[5] Norden, A. D., and Blumenfeld, H. 2002. "The Role of Subcortical Structures in Human Epilepsy.” Epil. Behav. 3: 219-31.

[6] Ziemann, A. E., et al. 2008. "Seizure Termination by Acidosis Depends on ASIC1a." Nat Neuro-Science 11: 816-22.

[7] Heiss, J. F., Katz, Y., Ganmor, E., and Lampl, L. 2008.
"Shift in the Balance between Excitation and Inhibition during Sensory Adaptation of SI Neurons.” $J$. Neuroscience 28: 13320-30.

[8] Cope, D. W., et al. 2009. "Enhanced Uonic GABA Inhibition in Typical Absence Epilepsy.” Nature Medicine 15: 1392-8.

[9] Hu, C., et al. 2018. "The Generation Mechanism of Spike and Slow Wave Discharges Appearing on Thalamic Delay Nuclei.” Sci Reports 8: 4953, 1-13.

[10] Jisra, V. K., Stacy, W. C., Quilichim, P. P., Ivanov, A. I., and Bernard, C. 2014. "On the Nature of Seizure Dynamics.” Brain 137: 2210-30.

[11] Von Neumann, J. 1966. Theory of Self-reproducing Automata, edited and completed by Arthur Burks, University of Illinois Press.

[12] Tsoutsouras, V., et al. 2012. "Simulation of Healthy and Epileleptiform Brain Activity Using Cellular Automata.” Journal of Bifurcation and Chaos 22: 1250229.

[13] Bragin, et al. 1999. "Hipocampal and Entorhinal Cortex High Frequency Oscillations in Human Epileptic Brain.” Epilepsia 40: 27-37.

[14] McCormick, D. A., and Contreras, D. 2001. "On the Cellular and Network Bases of Epileptic Seizures.” Annu. Rev. Physiol. 63: 815-46. 